\title{
COMPÓSITOS POLIMÉRICOS REFOÇADOS POR FIOS ALEATÓRIOS $E$ DESCONTÍNUOS DE JUTA: CARACTERIZAÇÃO MECÂNICA EM TRAÇÃO NA ASTM D638
}

\author{
G. M. NASCIMENTO ${ }^{1}$, R. S. M. DIAS ${ }^{1}$, I. S. GOMES ${ }^{1}$, L. C. O. PEREIRA ${ }^{1}$ e R. T. \\ FUJIYAMA $^{1}$
}

${ }^{1}$ Universidade Federal do Pará, Faculdade de Engenharia Mecânica

E-mail para contato: rodrigo.ma.galhaes@ hotmail.com

\begin{abstract}
RESUMO - Este trabalho apresenta os estudos de comparação de materiais compósitos de fibras de juta de 5 e $15 \mathrm{~mm}$ e resina poliéster na normas ASTM D638. A fibra de juta é encontrada com facilidade na região metropolitana de Belém, a partir do estudo da mesma, foi comprovado que sua utilização é viável na confecção de compósitos. Iniciou-se a fabricação do compósito sem nenhum tratamento prévio nas fibras. Foram fabricados os corpos de prova nas normas ASTM D638 para ambos os comprimentos de fibra natural com resina poliéster. Após a confecção dos corpos de prova, os mesmos passaram por processos de adequação as normas e logo em seguida encaminhados para o ensaio de tração para análise de suas propriedades mecânicas e seus resultados discutidos no decorrer do artigo. $\mathrm{O}$ estudo mostra que as melhores propriedades mecânicas foram obtidas pelas fibras de $15 \mathrm{~mm}$, onde a tensão média máxima foi de 21,17 $\mathrm{MPa}$, porém observou-se descontinuidades e desalinhamento das fibras na matriz, enquanto que nas de 5,0 $\mathrm{mm}$ estes resultados foram mais satisfatórios.
\end{abstract}

\section{INTRODUÇÃO}

De acordo com estudos de Ligosky (2015), a conscientização da sociedade firmada no conceito de desenvolvimento sustentável exige a produção de materiais ecológicos de baixo custo e consumo de energia reduzida. Buscam-se produtos capazes de satisfazer requisitos fundamentais como a conservação de recursos naturais não renováveis e a manutenção de um ambiente saudável. Os materiais compósitos, produzidos com fibras naturais, destacam-se neste conceito.

O uso de fibras vegetais como elemento de reforço em compósitos poliméricos é de grande interesse, pois estas provêm de fontes renováveis, são mais leves e custam menos do 


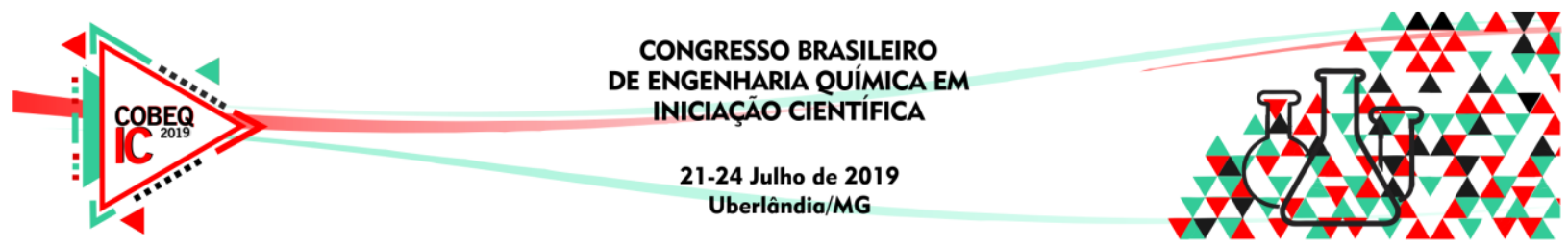

que as fibras sintéticas; são pouco abrasivas aos equipamentos de processo e não poluem o ambiente. As fibras naturais mais comuns usadas como reforços em polímeros são: juta, sisal, abacaxi e coco. Entre essas, a fibra de juta é uma das mais usadas como reforços de matrizes termoplásticas e termofixas, em virtude de seu baixo custo, abundância, maleabilidade e por apresentar, isoladamente, um bom conjunto de propriedades como, por exemplo, elevado módulo e resistência específica (NETO, 2007).

Este trabalho buscou analisar as propriedades mecânicas de compósitos de matriz polimérica de resina de poliéster reforçado por fibras de juta de 5,0 mm e 15,0 mm assistidos pela norma técnica ASTM D638. A partir de ensaios mecânicos de tração, busca-se avaliar o comportamento dos corpos de prova no que tange a rigidez do material, e, então, comparar os resultados dos módulos de elasticidade, tensão de ruptura dos compósitos ensaiados e aspectos da fratura dos mesmos.

\section{MATERIAIS E MÉTODOS}

\subsection{Materiais}

A matriz utilizada no desenvolvimento do compósito estudado neste trabalho foi a resina poliéster tereftálica insaturada, fabricada pela Royal Polímeros sob a denominação comercial de Denverpoly 754. O agente de cura utilizado foi o peróxido de MEK (Butanox M-50), nas proporções de $0,33 \%(v / v)$.

Como reforço, foram utilizadas fibras de juta de 5,0 $\mathrm{mm}$ e $15,0 \mathrm{~mm}$. A fabricação dos materiais se deu segundo a ASTM D638, produzindo-se, para tanto, dois moldes de silicone, os quais comportaram o formato dos corpos de provas sugeridos na norma (quatro para cada molde). Os materiais utilizados são, portanto, mostrados na Figura 1.

Figura 1: Materiais utilizados seguindo a metodologia de fabricação de corpos de prova. a) Fibras de juta de $5 \mathrm{~mm}$; b) Resina e acelerador de cura; c) Molde de silicone.

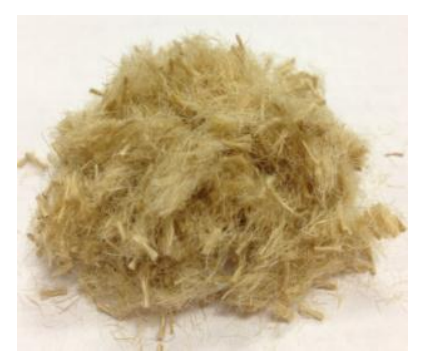

a)

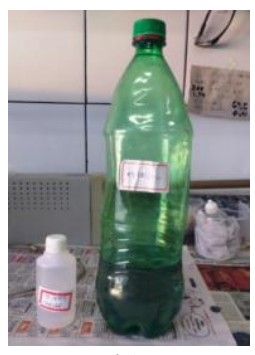

b)

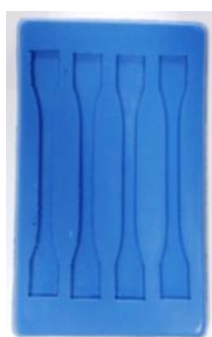

c)

\subsection{Métodos}

Primeiramente misturou-se a resina poliéster com a fibra de juta de $5 \mathrm{~mm}$ e $15 \mathrm{~mm}$ (separadamente para cada comprimento). Na fabricação dos corpos de prova na norma ASTM D638 utilizou-se os seguintes métodos: Deposição de fina camada de cera desmoldante, de 


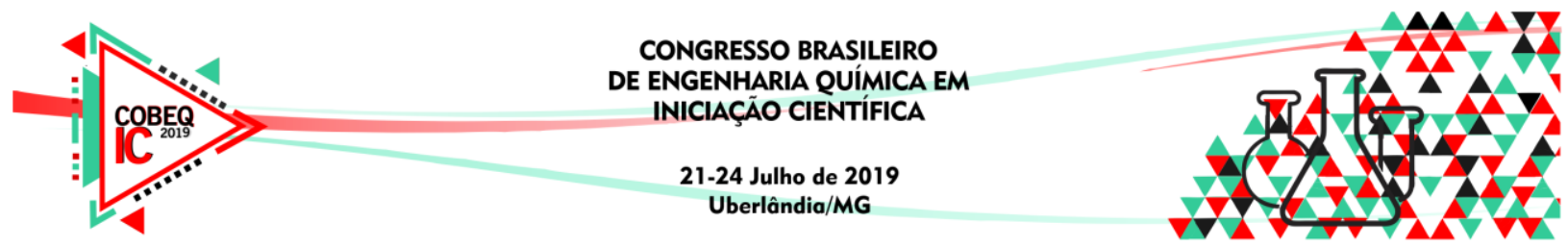

modo a facilitar a retirada dos corpos de prova; aferição da quantidade de fibra de juta adequada ao molde de silicone, mistura matriz/reforço e vazamento do material no molde silicone. Os moldes são capazes de produzir quatro corpos de prova.

Para a fabricação do material, utilizou-se o total de $61,5 \mathrm{~g}$ de resina poliéster tereftálica sem pré-aceleração com adição de $0,15 \mathrm{~g}$ do agente de cura peróxido de MEK (butanox m 50).

As fibras foram dispostas de forma aleatória no molde, facilitando assim sua dispersão na resina. A fração mássica apresentada para as fibras de $5 \mathrm{~mm}$ foram de $6,7 \%$ e para juta de $15 \mathrm{~mm}$ de $7,95 \%$.

Foram produzidos, então, em dois moldes de silicone quatro corpos de prova respectivamente para as fibras de 5,0 e $15,0 \mathrm{~mm}$, totalizando oito corpos de prova. A Figura 2 mostra as misturas conformadas sob os moldes de acordo com a norma técnica.

Figura 4 - Mistura fibra/resina disposta sob moldes de silicone. a) Fibras de 5,0 mm; b)Fibras de 15,0 mm.

a)

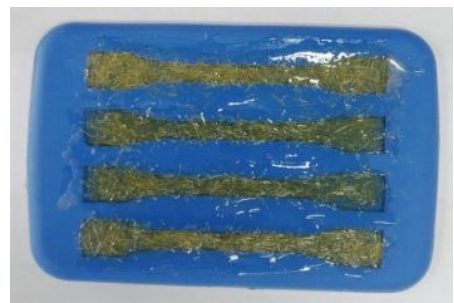

b)

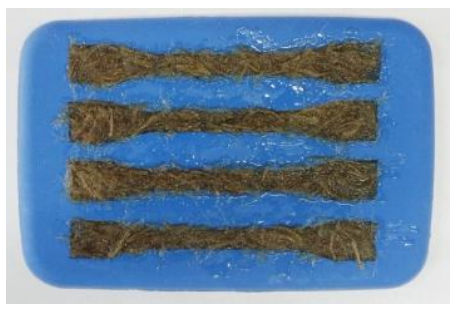

Os corpos de prova após desmoldados apresentam dimensões e superfícies irregulares, para solução desse problema é necessário o processo de lixamento para posteriormente serem marcados e submetidos ao ensaio mecânico. A Figura 3 exemplifica o processo descrito com os corpos de prova de juta de $15,0 \mathrm{~mm}$.

Figura 3 - Corpos de prova de juta $15 \mathrm{~mm}$ a) antes e b) depois do lixamento.

a)

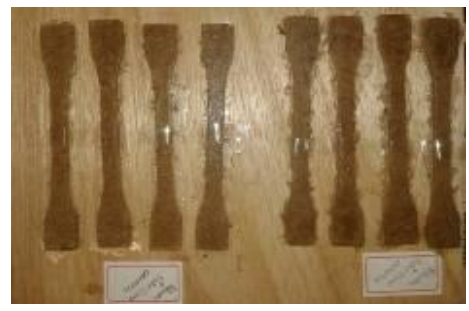

b)

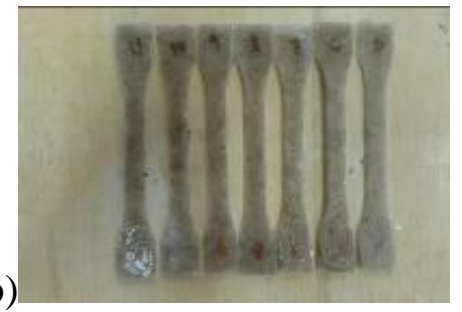

Após a fabricação, os corpos de prova foram levados para o ensaio de tração seguindo os procedimentos recomendados pela norma ASTM D638, em uma Máquina Universal de Ensaio (MUE) da marca Emic. O ensaio é mostrado na Figura 4. 


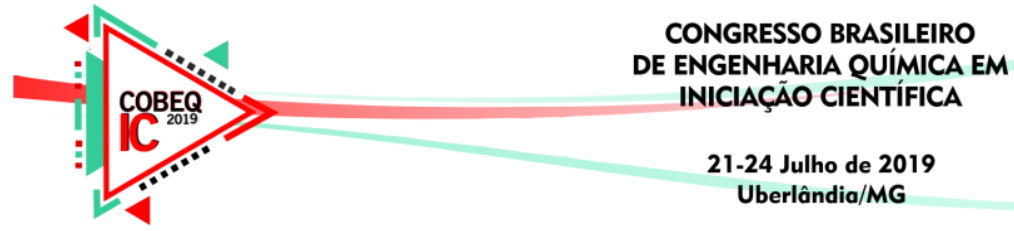

Figura 4 - Ensaio mecânico de tração para os corpos de prova fabricados com fibras naturais.

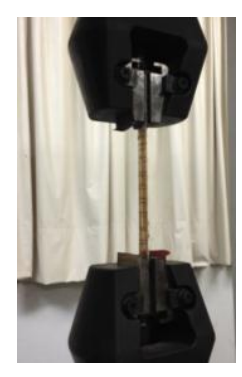

\section{RESULTADOS}

As propriedades mecânicas médias obtidas através dos ensaios de tração dos compósitos com reforço de fibras de 5,0 mm são apresentadas na Tabela 1.

Tabela 1 - Propriedades mecânicas em tração dos compósitos reforçados por fibras de juta de $5 \mathrm{~mm}$ e $15 \mathrm{~mm}$.

\begin{tabular}{|c|c|c|c|c|c|}
\hline $\begin{array}{c}\text { Tipo de } \\
\text { Material }\end{array}$ & $\begin{array}{c}\text { Fração Mássica de } \\
\text { Reforço }(\%)\end{array}$ & $\begin{array}{c}\text { Força Máxima } \\
(\mathrm{kN})\end{array}$ & $\begin{array}{c}\text { Tensão Máxima } \\
(\mathrm{MPa})\end{array}$ & $\begin{array}{c}\text { Deslocamento } \\
\text { Máximo }(\mathrm{mm})\end{array}$ & $\mathrm{E}(\mathrm{GPa})$ \\
\hline $\begin{array}{c}\text { Resina } \\
\text { Poliéster/Juta de } \\
5 \mathrm{~mm}\end{array}$ & 6,7 & $517,92 \pm 138,15$ & $17,59 \pm 4,69$ & $4,15 \pm 0,40$ & $0,230 \pm 0,12$ \\
\hline $\begin{array}{c}\text { Resina } \\
\text { Poliéster/Juta de } \\
15 \mathrm{~mm}\end{array}$ & 7,95 & $623,35 \pm 189,12$ & $21,17 \pm 6,42$ & $4,76 \pm 0,42$ & $0,210 \pm 0,98$ \\
\hline
\end{tabular}

Após a análise dos resultados, observa-se que a fibra de juta de $15 \mathrm{~mm}$ apresentou desempenho melhor para resistência mecânica com tensão média de 21,17 MPa, enquanto que o compósito com fibras de 5,0 $\mathrm{mm}$ apresentou 17,59 MPa. Com o aumento do comprimento das fibras, a atuação do reforço foi mais efetiva aumentando assim a resistência do compósito. Os dados indicam quem a resistência do compósito produzido aumentou com o aumento do comprimento da fibra, confirmando o que tem sido reportado por outros pesquisadores.

Nos compósitos com fibras curtas, a maior concentração de tensão ao longo da interface fibra/matriz ocorre nas extremidades das fibras e quanto menor o comprimento das fibras, maior o número destas extremidades, o que acarreta depreciação das propriedades mecânicas de tração do compósito (Silva, 2003).

De acordo com Bisaria (2015), a fibra de juta tem moderada resistência à tração e à flexão quando comparadas com outras fibras naturais. Os compósitos de polímero reforçados com fibra de juta são adequados para as aplicações estruturais primárias, elementos interiores 


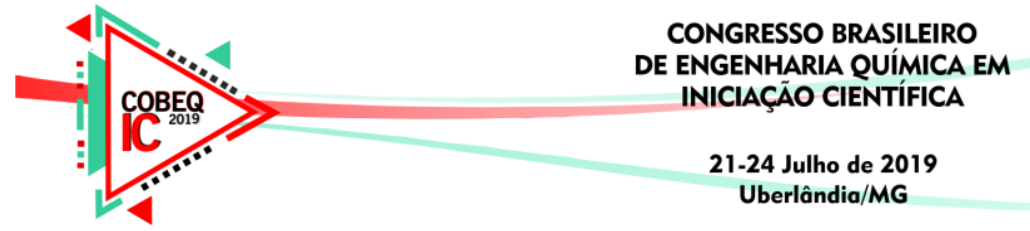

e aplicações temporárias ao ar livre. A característica de isolamento da juta torna-se útil em portas e teto automotivos, painéis de separação do motor e compartimentos de passageiros.

De modo geral, utilização de fibras vegetais em compósitos envolve todo um processo de consciência ambiental, social e econômico por materiais renováveis e de baixo custo. A Tabela 2 apresenta as propriedades mecânicas médias de compósitos poliméricos reforçados por fibras vegetais de $15 \mathrm{~mm}$ de acordo com o que cita a literatura comparados com .

Tabela 2 - Propriedades mecânicas em tração dos compósitos reforçados por fibras vegetais de $5 \mathrm{~mm}$ e $15 \mathrm{~mm}$ citados na literatura.

\begin{tabular}{|c|c|c|c|}
\hline Material compósito & Comprimento das fibras (mm) & Resistencia a tração (MPa) & Referência \\
\hline \hline \multirow{2}{*}{ Poliéster/Malva } & 5 & $20,42 \pm 1,53$ & COSTA et. al. (2013) \\
\cline { 2 - 4 } & 15 & $25,48 \pm 1,17$ & COSTA et. al. (2013) \\
\hline \multirow{2}{*}{ Poliéster/Piaçava } & 5 & $15,09 \pm 1,5$ & COSTA et. al. (2013) \\
\cline { 2 - 4 } & 15 & $19,56 \pm 1,32$ & COSTA et. al. (2013) \\
\hline \multirow{2}{*}{ Poliéster/Palha da costa } & 5 & $10,01 \pm 0,89$ & COSTA et. al. (2013) \\
\cline { 2 - 4 } & 15 & $16,48 \pm 1,31$ & BARBOZA (2013) \\
\hline
\end{tabular}

Dentre os compósitos reforçados por fibras vegetais, o de Poliéster/Malva foi o que apresentou maiores valores referentes à resistência a tração, sendo estes de 20,42 MPa paras as fibras de $5 \mathrm{~mm}$ e $25,48 \mathrm{MPa}$ para as de $15 \mathrm{~mm}$. Estes compósitos apresentaram valores de resistência a tração superior, se comparado com ao de Poliéster/Juta estudado neste trabalho, de $16,09 \%$ e $20,36 \%$ para as fibras de $5 \mathrm{~mm}$ e $15 \mathrm{~mm}$ respectivamente.

Os compósitos de Poliéster/Piaçava e Poliéster/Palha da costa apresentam menor resistência a tração em relação ao compósito estudado, haja vista que o primeiro apresenta uma diferença de $16,57 \%$ para as fibras de $5 \mathrm{~mm}$ e $8,23 \%$ para as de $15 \mathrm{~mm}$. A diferença em relação ao Poliéster/Palha da costa foi maior sendo esta de $75,72 \%$ para as fibras de $5 \mathrm{~mm}$ e $28,46 \%$ para as de $15 \mathrm{~mm}$.

\subsection{Análise da fratura dos materiais}

A Figura 4 mostra a superfície de um dos corpos de provas com fibras de 5,0 mm e 15,0 mm fraturadas. 


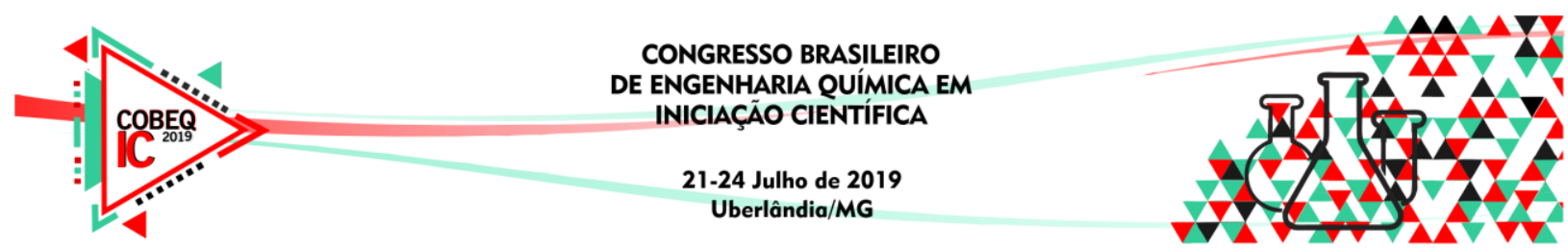

Figura 4 - Superfície fraturada dos corpos de prova após os ensaios de tração. a) Fibras de $5,0 \mathrm{~mm}$; b) Fibras de 15,0 mm.

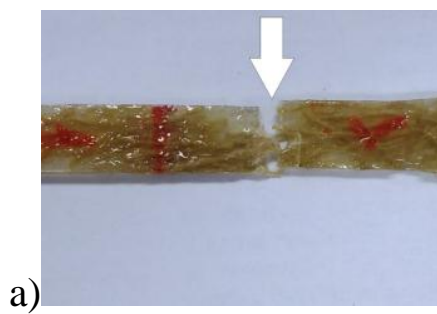

b)

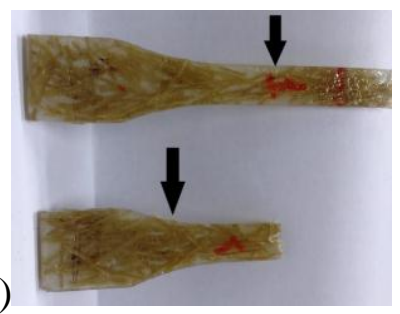

Observa-se a descontinuidade e o desalinhamento das fibras na matriz. É importante salientar que o compósito com fibras descontinua apresentam propriedades mecânicas menores que os de fibras contínuas devido ao fato de que a resistência e o reforço máximo são obtidos na direção do alinhamento, contudo, a vantagem da utilização de fibras descontinua consiste no menor custo de produção e uma optimização do tempo de fabricação, além das técnicas de fabricação que incluem processos manuais.

\section{CONCLUSÃO}

A utilização de fibras naturais em materiais compósitos representa uma nova forma de reutilização de matéria prima que outrora estaria sendo desperdiçada. A fibra de juta, utilizada como reforço neste projeto, em especial, apresentou bons desempenhos no processo de fabricação, utilizada in natura, sem tratamentos, mostrou ser possível sua fabricação em processo de laminação manual sem prejuízos para seu desempenho mecânico. Os ensaios de tração demonstraram que ocorre variação entre os valores obtidos em cada propriedade mecânica de acordo com o tamanho de fibras e norma utilizada. De modo geral os compósitos de fibra de juta e matriz poliéster apresentaram bons desempenhos mecânicos comparados aos valores de matriz pura, comprovando que a sua utilização é viável na fabricação de compósitos estruturais.

\section{REFERÊNCIAS}

BARBOSA, D. O.,Características de compósitos de fibras de palha da costa com resina poliéster. Belém, 34f. Trabalho de Conclusão de Curso (Engenharia Mecânica), Faculdade de Engenharia Mecânica, Universidade Federal do Pará, Belém, 2013.

BISARIA, H., GUPTA, M. K., SHANDILYA, P., SRIVASTAVA, R. K. Effect of fibre length on mechanical properties of randomly oriented short jute fibre reinforced epoxy composite. Materials Today: proceedings, v. 2, p.1193 - 1199, 2015.

COSTA, D, S.; EL BANNA, W. R.; SANTOS, E. J. S.; LOPES, C. E. P.; LIRA JUNIOR, P. F.; FUJIYAMA, R. T. Compósito de Poliéster com Fibras de Piaçava (Attalea funifera). In: 68 Congresso da ABM Internacional, 2013, Belo Horizonte. ABM, 2013. 


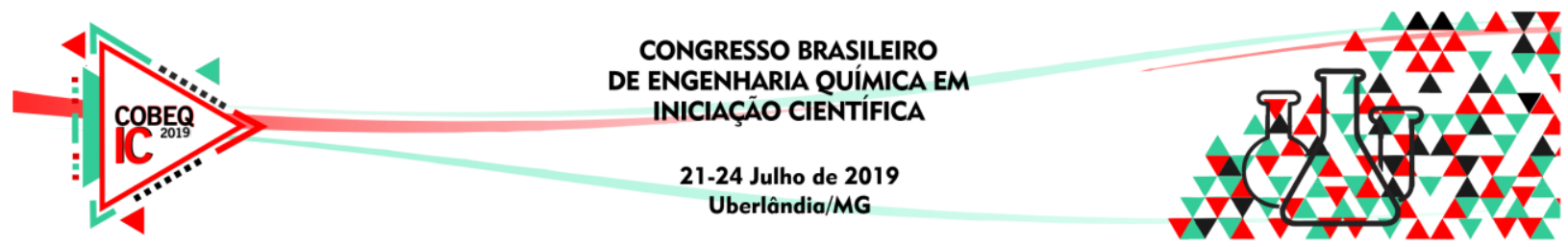

COSTA, D, S.; EL BANNA, W. R.; DE LIMA, L. S.; SANTOS, E. J. S.; LOPES, C. E. P.; FUJIYAMA, R. T. Materiais compósitos reforçados com fibras de malva. In: 68 Congresso da ABM Internacional, 2013, Belo Horizonte. ABM, 2013.

COSTA, D, S.; EL BANNA, W. R.; ALMEIDA, D. F.; SANTOS, E. J. S.; LOPES, C. E. P.; DE LIMA, L. S.; FUJIYAMA, R. T. Características de compósitos de fibras de palha da costa (Raphia vinífera) com resina de poliéster. In: 68 Congresso da ABM Internacional, 2013, Belo Horizonte. ABM, 2013.

GILDO, C. C. R., Compósito poliéster reforçado por fibras de sisal: avaliação do comprimento e proporção das fibras nas propriedades mecânicas dos compósitos. Belém, 36f. Trabalho de Conclusão de Curso (Engenharia Mecânica), Faculdade de Engenharia Mecânica, Universidade Federal do Pará, Belém, 2013.

LIGOWSKI, E., SANTOS, B. C., FUJIWARA, S.T. Materiais compósitos a base de fibras da cana-de-açúcar e polímeros reciclados obtidos através da técnica de extrusão. Revista Polímeros: Ciência e Tecnologia, vol.25, n1, p.70-75, 2015.

NETO, J. R. A., CARVALHO, L. H., ARAÚJO, E. M. (2007) "Influência da Adição de uma Carga Nanoparticulada no Desempenho de Compósitos Poliuretano/Fibra de Juta", Polímeros: Ciência e Tecnologia, vol. 17, nº 1, p. 10-15.

RODRIGUES, J. da S. Comportamento mecânico de material compósito de matriz poliéster reforçado por sistema híbrido fibras naturais e resíduos da indústria Madeireira. Belém: 2008, 91 f. Dissertação (Mestrado em Engenharia: Materiais e processos de Fabricação) - Faculdade de Engenharia Mecânica, Instituto de Tecnologia, Universidade Federal do Pará, Belém, 2008.

SILVA, R. V. Compósito de Resina Poliuretano Derivada de Óleo de Mamona e Fibras Vegetais. 2003. 157 f. Tese (Doutorado em Ciência e Engenharia dos Materiais) Universidade de São Paulo, São Paulo, 2003. 